$$
\begin{gathered}
\text { CONF-970510--4 } \\
\text { SAND97-0763C } \\
\text { SAND- }-97-0763 C
\end{gathered}
$$

\title{
Anomalous Normal Mode Oscillations in Semiconductor Microcavities
}

\author{
Hailin Wang \\ Department of Physics, University of Oregon, Eugene, OR 97403 \\ H.Q. Hou and B.E. Hammons \\ Sandia National Laboratories, Albuquerque, NM 87185 \\ 514-346-4758 (voice) \\ 541-346-4791 (fax)
}

Abstract

Transient four wave mixing studies reveal an anomalous normal mode oscillation when the cavity is tuned below the heavy hole exciton resonance. The result demonstrates the importance of virtual excitation for understanding optical properties of semiconductor microcavities.

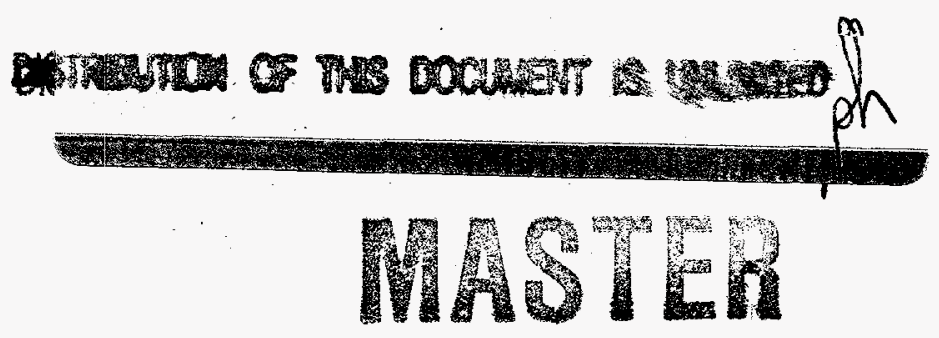




\section{DISCLAIMER}

This report was prepared as an account of work sponsored by an agency of the United States Government. Neither the United States Government nor any agency thereof, nor any of their employees, make any warranty, express or implied, or assumes any legal liability or responsibility for the accuracy, completeness, or usefulness of any information, apparatus, product, or process disclosed, or represents that its use would not infringe privately owned rights. Reference herein to any specific commercial product, process, or service by trade name, trademark, manufacturer, or otherwise does not necessarily constitute or imply its endorsement, recommendation, or favoring by the United States Government or any agency thereof. The views and opinions of authors expressed herein do not necessarily state or reflect those of the United States Government or any agency thereof. 


\section{DISCLADMER}

Portions of this document may be illegible in electronic image products. Images are produced from the best available original document. 


\title{
Anomalous Normal Mode Oscillations in Semiconductor Microcavities
}

\author{
Hailin Wang \\ Department of Physics, University of Oregon, Eugene, OR 97403 \\ H.Q. Hou and B.E. Hammons \\ Sandia National Laboratories, Albuquerque, NM 87185
}

Semiconductor microcavities as a composite exciton-cavity system can be characterized by two normal modes. Under an impulsive excitation by a short laser pulse, optical polarizations associated with the two normal modes have a $\pi$ phase difference. The total induced optical polarization is then expected to exhibit a $\sin ^{2}(\Omega t)$-like oscillation where $2 \Omega$ is the normal mode splitting, reflecting a coherent energy exchange between the exciton and cavity.

In this paper we present experimental studies of normal mode oscillations using threepulse transient four wave mixing (FWM). Our result reveals surprisingly that when the cavity is tuned far below the exciton resonance, normal mode oscillation in the polarization is $\cos ^{2}(\Omega t)$ like, in contrast to what is expected from the simple normal mode model. This anomalous normal mode oscillation reflects the important role of virtual excitation of electronic states in semiconductor microcavities.

The microcavity sample used consists of 16 and $22 \lambda / 4$ stacks of $\mathrm{Al}_{0.11} \mathrm{Ga}_{0.89} \mathrm{As} / \mathrm{AlAs}$ as the top and bottom mirrors, respectively. Four $130 \AA$ GaAs quantum wells are placed at the center of a $\lambda$ cavity. Small variations in the cavity thickness allow us to tune the cavity resonance by moving the laser spot across the sample while the exciton energy remains nearly unchanged. Figure 1 shows the dispersion of the two normal modes as we vary the detuning between the exciton and cavity. All measurements were performed at $10 \mathrm{~K}$.

The geometry for three-pulse transient FWM is shown in Fig. 2. Information on the temporal evolution of the induced optical polarization is obtained by measuring the intensity of the FWM signal as a function of the delay between the first two pulses. A fixed delay between the second and the third pulse also allows us to introduce a waiting period $\tau=\mathrm{t}_{3}-\mathrm{t}_{2}$ before we read out the information on the induced optical polarization. 
Wang et al., Anomalous Normal Mode...

Figure 3 shows the intensity of the FWM signal as a function of $t_{2}-t_{1}$ and at various fixed delay $t_{3}-t_{2}$. In this measurement, the cavity is tuned to $7 \mathrm{meV}$ below the heavy hole exciton resonance and the laser pulse (150 fs duration) is centered between the cavity and exciton resonances. For $t_{3}-t_{2}=0$, the FWM response exhibits a pronounced $\cos ^{2}(\Omega t)$-like oscillation and the frequency of the oscillation agrees with the normal mode splitting. The phase of the oscillation changes rapidly as we vary the fixed delay $t_{3}-t_{2}$. With increasing fixed delays, the oscillation reverts to $\sin ^{2}(\Omega \mathrm{t})$-like as shown in Fig. 3.

To understand the above result, we first point out that the use of a fixed delay $t_{3}-t_{2}$ can separate out optical excitations with different life times. It is evident that $\sin ^{2}(\Omega t)$-like oscillations observed at long fixed delays are due to optical excitations with a relatively long life time. Similarly, $\cos ^{2}(\Omega \mathrm{t})$-like oscillations observed at nearly zero fixed delays can be considered as due to optical excitations with a very short life time (between 1 and $2 \mathrm{ps}$ ).

A likely mechanism for excitations with such an extremely short life time is virtual excitation of electronic states away from heavy hole excitons (i.e. light hole excitons and continuum states). The life time of a virtual excitation is determined by the duration of the optical pulse and in this case the cavity life time, which is approximately 1.5 ps for our sample. The virtual excitation contributes to the coherent nonlinear optical response through processes such as optical Stark effects. Optical polarization induced by the virtual excitation is also expected to follow the applied field and hence to exhibit a $\cos ^{2}(\Omega t)$-like oscillation. This further suggests that in general the phase of the normal mode oscillation can be used as an effective probe for nonlinear optical response in composite exciton-cavity systems.

This work is supported by AFOSR.

Sandia is a multiprogram laboratory operated by Sandia Corporation, a Lockheed Martin Company, for the United States Department of Energy under Contract DE-ACO4-94AL85000. 
Figure Captions:

Fig. 1 Energy positions of the two normal modes as we vary the detuning between the exciton and cavity by moving the laser spot across the sample.

Fig. 2 Experimental geometry for the three-pulse transient FWM. The signal is along the direction of $\mathbf{k}_{1}-\mathbf{k}_{2}+\mathbf{k}_{3}$.

Fig. 3 FWM response as a function of $t_{2}-t_{1}$ and at various fixed delay $\tau=t_{3}-t_{2}$. Notice a $\pi$ phase change as we increase fixed delays. 
Wang et al., Anomalous Normal Mode...

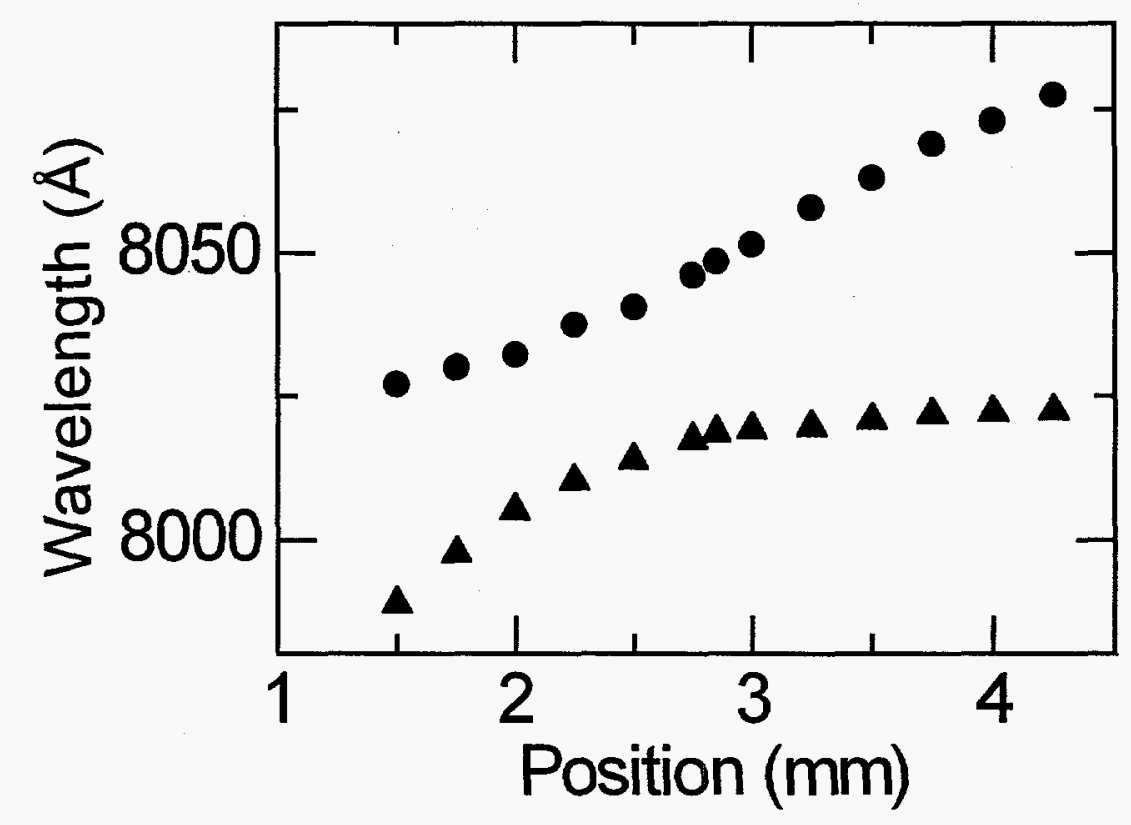

Fig. 1 

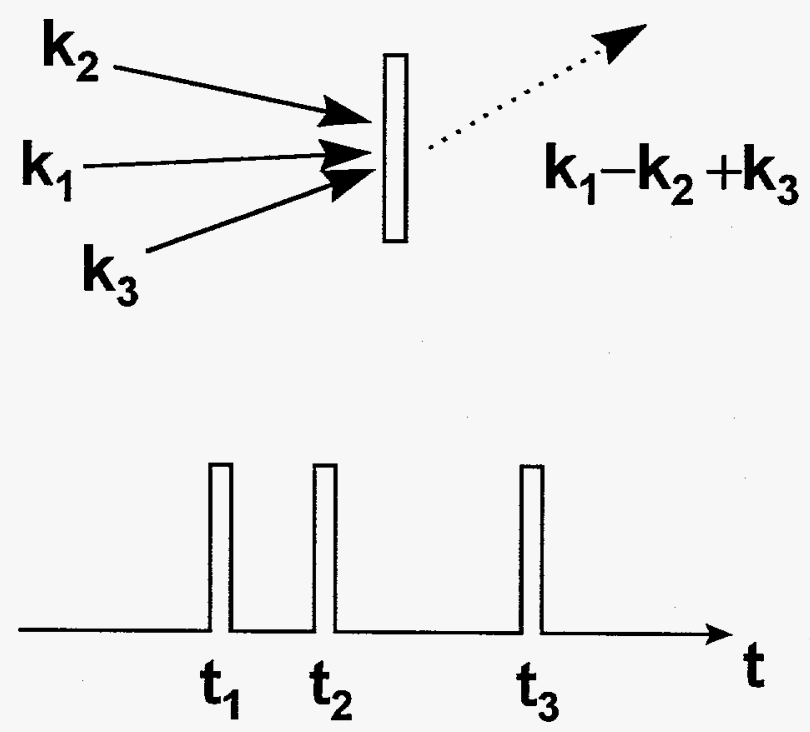

Fig. 2 
Wang et al., Anomalous Normal Mode...

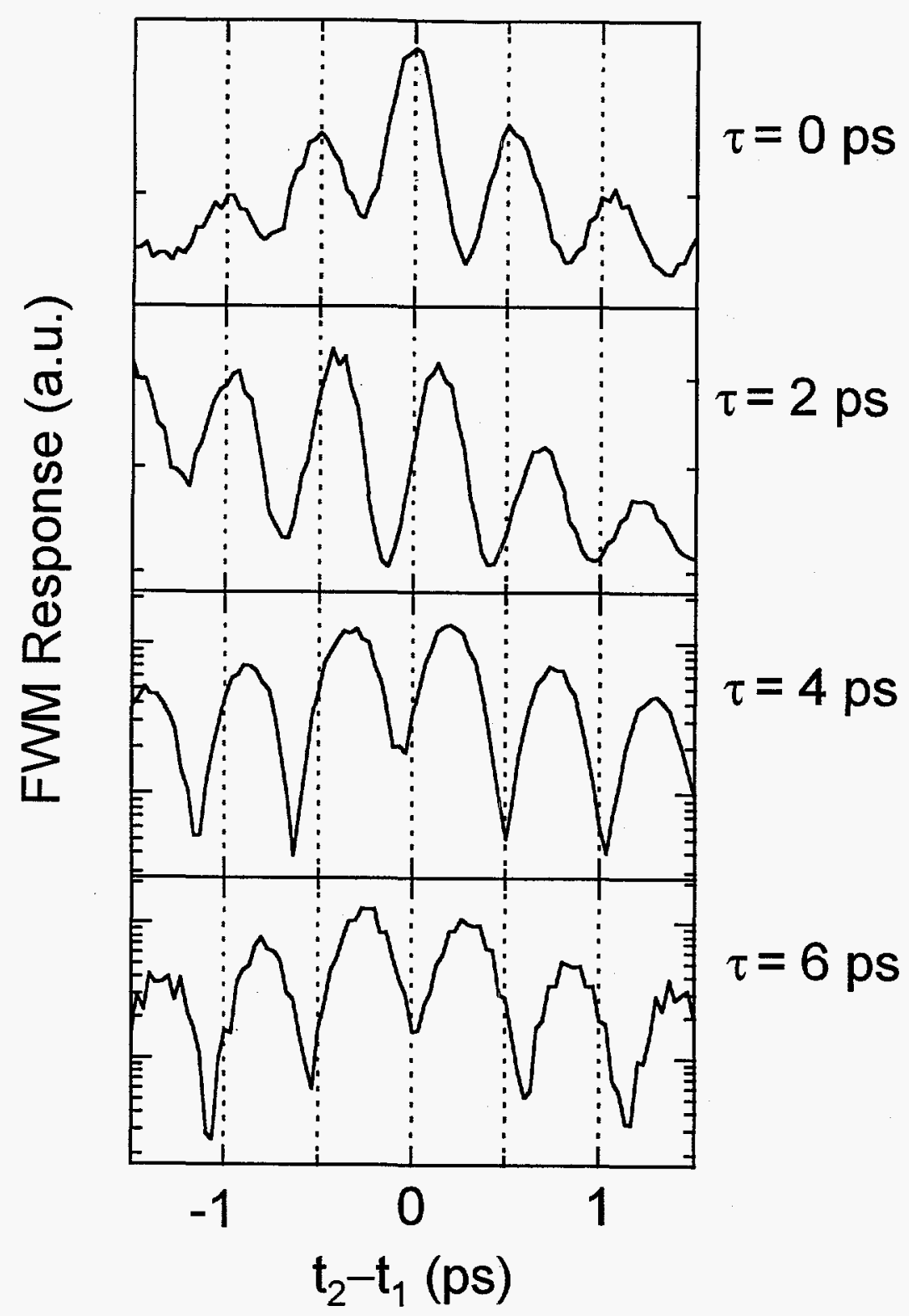

Fig. 3 